\title{
Simulation of Acoustical Properties of Coupled Rooms Using Numerical Technique Based on Modal Expansion
}

\author{
M. MEISSNER \\ Institute of Fundamental Technological Research, Polish Academy of Sciences \\ Adolfa Pawińskiego 5B, 02-106 Warsaw, Poland
}

\begin{abstract}
In the present study, the acoustic characteristics of coupled rooms were investigated using the computer modelling application based on the modal expansion method. A numerical procedure enables the computation of shape and frequency of eigenmodes, and allows one to predict sound decay times at any point of a room space. Results of numerical simulation have shown that along with a change in a coupling area the effect of a mode degeneration appeared and there are two main reasons for this. First, a mode degeneration is associated with a convergence of frequencies of neighbouring modes with a decrease of coupling area. Another reason for the mode degeneration are variations in a sequence of modes with a change in the coupling area. It was demonstrated that in the coupled rooms several modes exhibit a considerable localization. A degree of mode localization was described theoretically by the "existence surface" of mode and it was found that the localization phenomenon has a great effect on the reverberation process because for some absorbing material distributions it caused the double-slope decay characterized by steep initial and slow late sound decays.
\end{abstract}

PACS numbers: 43.55.Br, 43.55.Ka, 43.20.Ks

\section{Introduction}

Acoustical properties of coupled rooms have long been investigated in the context of architectural acoustics [1-4] because several closed spaces actually consist of smaller partial rooms which are coupled together. The orchestra pit and balconies in opera houses or theatres coupled to the main floor as well as churches with several naves and chapels are typical examples of architectural objects having a structure of coupled rooms. In order to obtain better understanding and control of acoustics in such room systems it is vital to have an efficient theoretical or computational method for predicting a structure of acoustic field in coupled rooms.

The geometric theory [5], the modal expansion method, known also as the modal analysis [6], ray-tracing techniques [7], and statistical or diffusion models [8,9] are main methods for determining the acoustics of irregularly shaped rooms. However, the geometric theory at best applies to enclosures with dimensions large compared to the wavelength. Moreover, this method neglects diffraction phenomena since a propagation in straight lines is its main postulate. A theory more adequate from the physical point of view but more difficult is the modal analysis because it bases upon the wave acoustics. The wave approach can be used in a low-frequency range, thus this theory has a practical application for enclosures having dimensions comparable with the sound wavelength.

In the modal expansion method the response of a room to a harmonic excitation can be understood in terms of its normal modes and the associated decay constant of each of these modes [10]. For small sound absorption on room walls the coupling between modes may be neglected and a distribution of mode amplitude can be well described by the eigenfunction for rigid room walls [11]. As distinct from regular rooms, such rectangular, triangular or cylindrical ones, in coupled rooms the amplitudes of rigid-walled modes and their frequencies are not definable analytically, therefore an application of modal analysis to such room systems was possible through numerical methods.
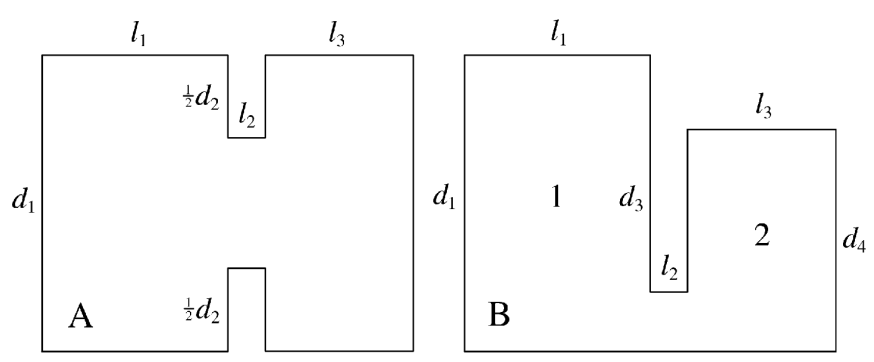

Fig. 1. Horizontal cross-sections of analyzed coupledroom systems A and B.

The subject of the present paper is a numerical simulation of acoustical properties of two-room coupled systems having the same height $h$, but different shape of horizontal cross-sections (Fig. 1). Proportions of room dimensions are the following: $l_{1} / l=0.5, l_{2} / l=0.1, l_{3} / l=0.4$, $d_{1} / l=0.8, d_{2} / d_{1}=0-0.95, d_{3} / l=0.64, d_{4} / l=0.6$ and $h / l=0.3$, where $l=l_{1}+l_{2}+l_{3}$. In the room system denoted by $\mathrm{A}$ the effect of a mode degeneration occurring due to a change in a coupling area was investigated. 
In the room system B consisting of two rectangular subrooms (in Fig. 1 denoted by 1 and 2) a phenomenon of mode localization and the effect of double-slope sound decay were analyzed. In a simulation program, the modal expansion for weakly damped rooms and the numerical technique being an extension of computational methods presented by the author in papers [12-14] were used.

\section{Theoretical background and numerical method}

A description of acoustical field in coupled rooms, having dimensions comparable with a length of acoustic wave, is based of a solution of the wave equation with specified initial and boundary conditions [10]. In this case a reverberation behaviour of room can be understood as a decay of normal acoustic modes determined by eigenfunctions $\Phi_{m n}(\boldsymbol{r})$ and eigenfrequencies $\omega_{m n}$, where $\boldsymbol{r}=(x, y, z)$ is a position vector and $m=0,1,2,3 \ldots$ and $n=0,1,2,3 \ldots$ When a source term in the wave equation has the form $-q(\boldsymbol{r}) \cos (\omega t)$, where $q(\boldsymbol{r})$ and $\omega$ are a spatial distribution and a frequency of source, the expression describing temporal decay of sound pressure can be written as [12]

$$
\begin{aligned}
& p(\boldsymbol{r}, t)=-\frac{Q_{00} \mathrm{e}^{-2 r_{00} t}}{\omega^{2}+4 r_{00}^{2}}+\sqrt{V} \sum_{m=0}^{\infty} \sum_{n=0}^{\infty} \\
& \frac{\xi_{m n} Q_{m n} \omega_{m n} \mathrm{e}^{-r_{m n} t} \cos \left(\Omega_{m n} t-\alpha_{m n}\right) \Phi_{m n}(\boldsymbol{r})}{\Omega_{m n} \sqrt{\left(\omega_{m n}^{2}-\omega^{2}\right)^{2}+4 r_{m n}^{2} \omega^{2}}},
\end{aligned}
$$

where $\xi_{00}=0$ and $\xi_{m n}=1$ for the other values of $m$ and $n, V$ is a room volume, $Q_{00}$ and $Q_{m n}$ are factors determining a sound source intensity

$$
\begin{aligned}
Q_{00} & =\frac{c^{2}}{V} \int_{V} q(\boldsymbol{r}) \mathrm{d} v, \\
Q_{m n} & =\frac{c^{2}}{\sqrt{V}} \int_{V} q(\boldsymbol{r}) \Phi_{m n}(r) \mathrm{d} v,
\end{aligned}
$$

where $c$ is a sound speed, $r_{00}$ and $r_{m n}$ are modal damping coefficients

$$
\begin{aligned}
& r_{00}=\frac{\rho c^{2}}{V} \int_{S} Z^{-1} \mathrm{~d} s, \\
& r_{m n}=\frac{\rho c^{2}}{2} \int_{S} \Phi_{m n}^{2}(\boldsymbol{r}) / Z \mathrm{~d} s,
\end{aligned}
$$

where $\rho$ is an air density, $S$ is a surface of room walls and $Z$ is a wall impedance. The phase shift $\alpha_{m n}$ in Eq. (1) is given by

$$
\alpha_{m n}=\tan ^{-1}\left[\frac{r_{m n}\left(\omega_{m n}^{2}+\omega^{2}\right)}{\Omega_{m n}\left(\omega_{m n}^{2}-\omega^{2}\right)}\right],
$$

where $\Omega_{m n}=\sqrt{\omega_{m n}^{2}-r_{m n}^{2}}$ is the eigenfrequency for damped oscillations. The functions $\Phi_{m n}$ depend on a room shape and are mutually coupled through the impedance condition on absorbing walls, but for low frequencies, where materials are characterized by a low absorption $(\Re e(Z / \rho c) \ll 1)$, it is possible to assume that a distribution of mode amplitude is well approximated by uncoupled eigenfunctions computed for perfectly rigid room walls [11]. For rooms having a constant height these eigenfunctions can be determined by

$$
\Phi_{m n}(\boldsymbol{r})= \begin{cases}\Psi_{n}(x, y) / \sqrt{h}, & m=0, \\ \sqrt{2 / h} \cos (m \pi z / h) \Psi_{n}(x, y), & m>0 .\end{cases}
$$

where functions $\Phi_{m n}$ are normalized in the room volume $V, h$ is the room height and eigenfunctions $\Psi_{n}$ are normalized over the surface $S_{0}$ of a room horizontal cross-section and $\Psi_{0}=1 / \sqrt{S_{0}}$. In this case eigenfrequencies $\omega_{m n}$ are given by

$$
\omega_{m n}=\sqrt{(m l / h)^{2}+\omega_{n}^{2}},
$$

where $\omega_{n}$ is a non-dimensional eigenfrequency corresponding to the function $\Psi_{n}$ and $\omega_{0}=0$. For the analysed room systems eigenfunctions $\Psi_{n}$ and eigenfrequencies $\omega_{n}$ were calculated numerically by the use of the forced oscillator method [15] with a finite difference algorithm. This method is based on the principle that a response of linear system to a periodic excitation is large when a driving frequency is close to a frequency of eigenmode.

\section{Acoustical behaviour of coupled rooms}

Using the numerical procedure, some interesting aspects of the acoustics in coupled rooms have been investigated. For the room system A (Fig. 1) it was analysed the occurrence of mode degeneration due to a change in the coupling area. The effect of mode localization was investigated in the room system B by means of the so-called "existence surface" of mode which characterizes an irregular distribution of mode amplitude inside a room space. It was shown that the mode localization has a great influence on the reverberation process because for some absorbing material distributions it causes the double-slope sound decay.

\subsection{Mode degeneration}

Calculation data shown in Fig. 2 depict changes in the non-dimensional eigenfrequency $\Omega_{n}=\omega_{n} / \omega_{r}$ with the parameter $\Delta / d_{1}$ for mode numbers $n$ of $11-13$ and 19-22, where $\omega_{r}=\pi c / l$ is a fundamental resonance frequency of the room system $\mathrm{A}$ and $\Delta=d_{1}-d_{2}$ is a measure of size of the coupling area. For modes 12 and 13 one can observe a very interesting behaviour of eigenmode frequency, namely a coincidence of frequencies of neighbouring modes with a decrease of coupling area (Fig. 2a). Explanation of this phenomenon can be found in Fig. 3 presenting shapes of eigenfunctions $\Psi_{n}$ for modes 12 and 13 for three different values of the non-dimensional parameter $\Delta / d_{1}$. The plots in Fig. 3 are in a form of filled contour maps, which are a two-dimensional representation of three-dimensional data, where contours define lines of constant value of $\Psi_{n}$. As $\Delta / d_{1}$ decreases, frequencies of these modes approach each other and finally, for value of $\Delta / d_{1}$ close to zero they become a pair of degenerate eigenmodes. Since an energy of these modes is concentrated in the first or the second part of a room, 
they represent also a pair of localized eigenmodes. The graphs in Fig. 2 also indicate that a change in a coupling area can force such variations of eigenmode frequency that changes, in a sequence of neighbouring modes on a frequency axis, occur. Consequently, the curves obtained for some neighbouring eigenmodes intersect, thus for values of $\Delta / d_{1}$ corresponding to intersection points there exist pairs of degenerate eigenmodes.

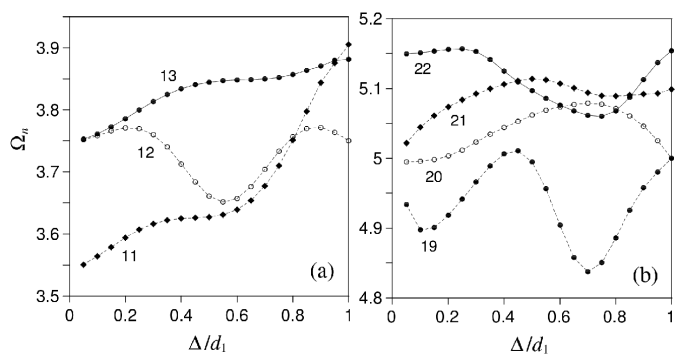

Fig. 2. Dependence of non-dimensional eigenfrequency $\Omega_{n}$ on parameter $\Delta / d_{1}$ for mode numbers $n$ : (a) 11-13, (b) 19-22.

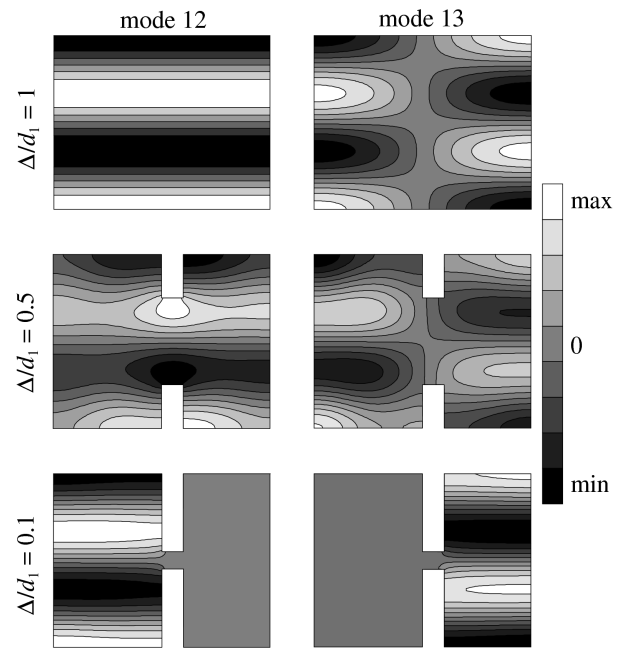

Fig. 3. Shapes of eigenfunctions $\Psi_{n}$ for modes 12 and 13 for parameter $\Delta / d_{1}$ equal to $0.1,0.5$ and 1 .

\subsection{Localization of modes}

In coupled rooms there exists a physical mechanism, namely mode localization, that for some eigenmodes creates such a distribution of mode amplitude that its energy is concentrated in a small part of a room space. This effect is a direct result of an irregular room geometry because in a rectangular room all eigenmodes are delocalized. In coupled enclosures having a constant height, the mode localization is a consequence of a complex shape of room horizontal cross-section. Thus, this phenomenon is connected with an irregular distribution of functions $\Psi_{n}$ in $(x, y)$ plane hence it may be treated as two-dimensional problem. In this case the mode localization can be characterized by the "existence surface" of mode defined as [16]

$$
S_{n}=\left[\int_{S_{0}} \Psi_{n}^{4}(x, y) \mathrm{d} x \mathrm{~d} y\right]^{-1} .
$$

In accordance with this definition, an eigenmode can be treated as being localized whenever the relative existence surface $S_{n} / S_{0}$ is found to be significantly smaller than unity. For example, in a rectangle, where all eigenmodes are delocalized, the parameter $S_{n} / S_{0}$ equals unity for Helmholtz mode, $2 / 3$ for axial modes and $4 / 9$ for oblique modes. In Fig. 4 is depicted a dependence of the relative existence surface on the non-dimensional eigenfrequency $\Omega_{n}$ and as may be seen, the irregular shape of room system $\mathrm{B}$ generates a considerable mode localization because for several modes the parameter $S_{n} / S_{0}$ has a value less than 0.3. Nearly twice as small value of $S_{n} / S_{0}$ is found for mode $37\left(\Omega_{n} \approx 7.55\right)$, for which the highest degree of mode localization is noted. Examples of computed shapes of function $\Psi_{n}$, for strongly localized eigenmodes, are shown in Fig. 5. From these plots it can be concluded that a high mode localization is accompanied with a concentration of mode energy in the first or the second subroom. This property has a practical aspect because the localization effect is of great importance in the process of sound decay, when there is a large difference between the absorption coefficient of walls in various parts of the room. In this case for frequencies of these eigenmodes, which are localized in the part of room where the sound absorption is much smaller, the non-linear decay of a sound pressure level may occur [17].

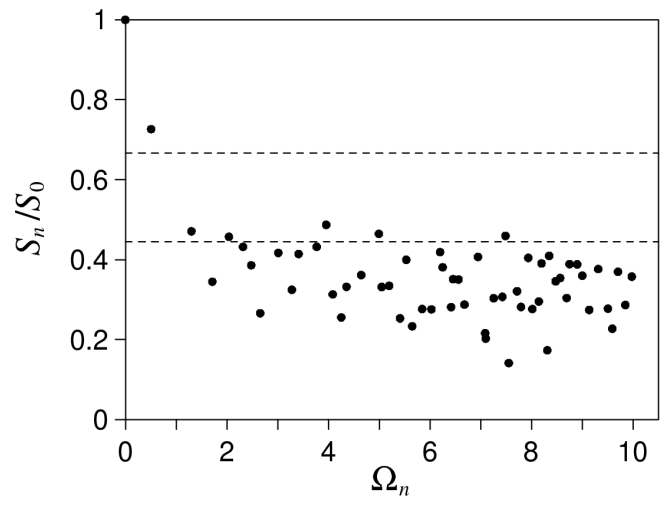

Fig. 4. Relative existence surface $S_{n} / S_{0}$ versus nondimensional frequency $\Omega_{n}$ for coupled-room system B (dots). Horizontal lines at $2 / 3$ and $4 / 9$ correspond to values of $S_{n} / S_{0}$ for rectangle.

\subsection{Double-slope sound decay}

For a given frequency, a position and a distribution of sound source, Eqs. (1)-(4) enable to predict the reverberation time at each point of room system B from calculated energy decay curves corresponding to a time history of the sound pressure level. In a computational 


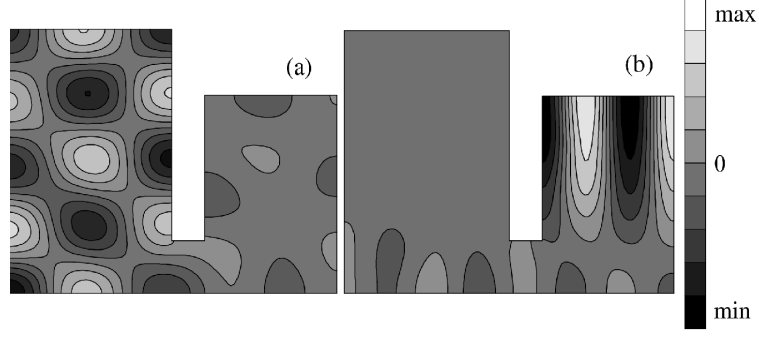

Fig. 5. Shapes of eigenfunctions $\Psi_{n}$ for mode numbers: (a) $n=27$, (b) $n=37$.

simulation it was assumed that the sound source is located in the subroom 1, whereas the observation point lies in the subroom 2. For a sake of model simplicity, the wall impedance $Z$ was assumed to be purely real, i.e. the mass and stiffness of the absorbing material are neglected. This corresponds to a damping of a sound wave on the room wall with no phase change upon a reflection. In order to examine an influence of walls absorption on the process of sound decay, in numerical analysis it was assumed that the random-absorption coefficient $\alpha_{1}$ of material on walls in the subroom 1 and the random-absorption coefficient $\alpha_{2}$ of material on walls in the subroom 2 were selected in this way, so that the total room absorption $A=\bar{\alpha} S$ remained constant, where $\bar{\alpha}$ is the average random-absorption coefficient of materials on room walls. In the numerical simulation it was assumed that $\bar{\alpha}=0.15$, thus the coefficients $\alpha_{1}$ and $\alpha_{2}$ were changing quantities. Since Eq. (1) for the sound pressure includes harmonic terms, the regression method was used to compute time-average decay curves of the pressure level $L$. To obtain enough data for a regression analysis, temporal changes in the pressure level were determined with the time interval $10^{-4} \mathrm{~s}$ and calculations were performed as long as a drop of the pressure level $L$ was smaller than $120 \mathrm{~dB}$.

The process of a creation of the double-slope decay is illustrated in Fig. 6, where pressure level decays (black lines) in one observation point together with fit curves (white lines) obtained for a sound frequency equal to the eigenfrequency of mode 37 and different absorption coefficients $\alpha_{1}$ and $\alpha_{2}$ in the first and the second subrooms are shown. When values of $\alpha_{1}$ and $\alpha_{2}$ are equal, a fit curve obtained via the regression method demonstrates a linear dependence on the time (Fig. 6a) and it means that a sound pressure has an exponential decay. For growing difference between $\alpha_{1}$ and $\alpha_{2}$ fit curves become more and more non-linear (Fig. 6b,c) and for the largest difference between absorption coefficients the fit curve consists of two parts referring to the rapid early decay and the slow late decay (Fig. 6d), which constitutes the double-slope decay of a pressure level.

A steep initial sound decay may result in higher sound clarity, whereas a slow late decay leads to an increase in perceived reverberation [18], thus from a subjective

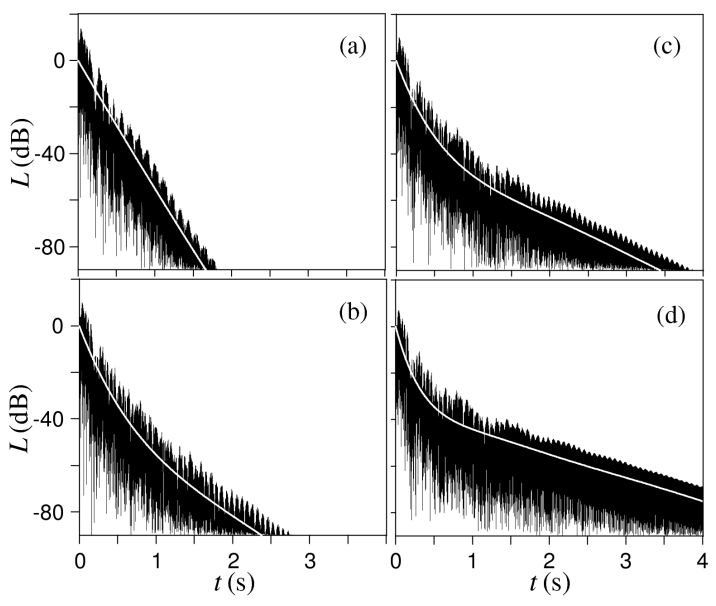

Fig. 6. Sound pressure level (black lines) and decay curves obtained via regression method (white lines) for absorption coefficients: (a) $\alpha_{1}=\alpha_{2}=0.15$, (b) $\alpha_{1}=0.22, \alpha_{2}=0.046$, (c) $\alpha_{1}=0.23, \alpha_{2}=0.031$, (d) $\alpha_{1}=0.24, \alpha_{2}=0.016$. Source frequency equal to frequency of mode 37 .

viewpoint the standard reverberation time (the time for a sound to die away to a level $60 \mathrm{~dB}$ below its original level) appears to misleading measure of the double-slope decay. In this case, to quantify decay profiles accurately, the early decay time (EDT) and the late decay time (LDT) should be computed, where EDT is $60 \mathrm{~dB}$ decay time calculated by a line fit to a portion of decay curve between 0 and $-10 \mathrm{~dB}$ and LDT is $60 \mathrm{~dB}$ decay time predicted from the decay between -50 and $-60 \mathrm{~dB}$.

\section{Conclusions}

In low-frequency range the coupled rooms exhibit some interesting effects like: a mode degeneration, a confinement of acoustic vibrations in a small part of room system, known as the localization of modes, and a considerable difference between a rate of sound decay in early and late stages of a reverberant process, which leads to the so-called double-slope sound decay. In this study, such effects were investigated using a simulation procedure based on the classical modal expansion. In a theoretical model it was assumed that a room system is lightly damped so a pressure variable was expanded in "hard box modes" that is, the normal modes for which boundary conditions are rigid walls. Eigenfunctions were computed numerically via application of the forced oscillator method with a finite difference algorithm. Results of computer simulation have shown that along with a change in a coupling area the effect of a mode degeneration appeared and there are two main reasons for this. Firstly, the degeneracy is connected with a gradual convergence of frequencies of neighbouring modes with a decrease of coupling area and this kind of mode degeneration is associated with the localization of modes. Another reason for the mode degeneration are variations in a se- 
quence of modes with a modification of a coupling area. In this case, frequencies of modes are equal to each other just before a change in a sequence of modes. A degree of mode localization was characterized mathematically by the "existence surface" of mode and it was found that as opposed to rectangular rooms, in coupled rooms several eigenmodes reveal a considerable localization. As was shown, the localization of modes has a great influence on a reverberation behaviour in coupled rooms because for some absorbing material distributions it caused the double-slope decay characterized by rapid early and slow late sound decays, thus, the curve describing a decay of sound intensity exhibited the so-called "sagging" appearance.

\section{References}

[1] J.S. Anderson, M. Bratos-Anderson, P.J. Donay, J. Sound Vib. 208, 313 (1997).

[2] J.S. Anderson, M. Bratos-Anderson, J. Sound Vib. 236, 209 (2000).

[3] A. Magrini, L. Magnani, Build. Acoust. 12, 115 (2005).

[4] M. Ermann, M. Johnson, J. Sound Vib. 284, 915 (2005).
[5] H. Kuttruff, Room acoustics, Applied Science Publishers, London 1973.

[6] P.M. Morse, R.H. Bolt, Rev. Mod. Phys. 16, 69 (1994).

[7] J.E. Summers, R.R. Torres, Y. Shimizu, B.L. Dalenbäck, J. Acoust. Soc. Amer. 118, 1491 (2005).

[8] J.E. Summers, R.R. Torres, Y. Shimizu, J. Acoust. Soc. Amer. 116, 958 (2004).

[9] A. Billon, V. Valeau, A. Sakout, J. Picaut, J. Acoust. Soc. Amer. 120, 2043 (2006).

[10] P.M. Morse, K.U. Ingard, Theoretical acoustics, McGraw-Hill, New York 1968.

[11] E. Dowell, G. Gorman, D. Smith, J. Sound Vib. 52, 519 (1977).

[12] M. Meissner, Arch. Acoust. 31, 239 (2006).

[13] M. Meissner, Cent. Eur. J. Phys. 5, 293 (2007).

[14] M. Meissner, Appl. Acoust. 69, 583 (2008).

[15] T. Nakayama, K. Yakubo, Phys. Rep. 349, 239 (2001).

[16] S. Russ, B. Sapoval, O. Haeberlé, Phys. Rev. E 55, 1413 (1997)

[17] M. Meissner, Arch. Acoust. 32, 213 (2007).

[18] D. Bradley, L. Wang, J. Acoust. Soc. Amer. 118, $1480(2005)$. 